\title{
Diagnosis of pneumothorax in critically ill adults
}

\author{
James J Rankine, Antony N Thomas, Dorothee Fluechter
}

\begin{abstract}
The diagnosis of pneumothorax is established from the patients' history, physical examination and, where possible, by radiological investigations. Adult respiratory distress syndrome, pneumonia, and trauma are important predictors of pneumothorax, as are various practical procedures including mechanical ventilation, central line insertion, and surgical procedures in the thorax, head, and neck and abdomen. Examination should include an inspection of the ventilator observations and chest drainage systems as well as the patient's cardiovascular and respiratory systems.

Radiological diagnosis is normally confined to plain frontal radiographs in the critically ill patient, although lateral images and computed tomography are also important. Situations are described where an abnormal lucency or an apparent lung edge may be confused with a pneumothorax. These may arise from outside the thoracic cavity or from lung abnormalities or abdominal viscera inside the chest. (Postgrad Med f 2000;76:399-404)
\end{abstract}

Keywords: pneumothorax; adult respiratory distress syndrome; critical care; radiography

In critical illness the diagnosis of pneumothorax is often complicated by other disease processes and by difficulties in imaging sick and unconscious patients. This article discusses the pathophysiology of pneumothorax and then describes the clinical and radiological diagnosis. Some examples of difficulties surrounding the radiological diagnosis of pneumothorax are then presented. The subject is important because pneumothorax is common in ventilated critically ill patients and failures in diagnosis can cause life threatening complications.

Hope Hospital, Salford, UK: Department of Radiology J J Rankine

Department of Intensive Care A N Thomas D Fluechter

Correspondence to: Dr James J Rankine, Department of Radiology, St James's University Hospital, Beckett Street, Leeds LS9 7TF, UK

Submitted 7 July 1999 Accepted 27 October 1999

\section{Pathophysiology}

The pleural space is the area between the parietal pleura on the inner surface of the chest wall and the visceral pleura on the outer surface of the lung. The space normally contains only a very small volume of fluid that allows the parietal and visceral pleura to move smoothly over each other. The chest wall and lung are both elastic in nature, with the lung tending to recoil inwards and the chest wall to spring outwards. These two opposing forces produce a negative (subatmospheric) pressure in the pleural space. The weight of the lung tends to make this pressure less negative in the dependent areas of the lung. If the pleural space is opened to the atmosphere then the subatmospheric pressure will suck air into the pleural space. The lung

\author{
Box 1: Mechanisms of air entry \\ causing pneumothorax \\ - Chest wall damage: \\ Trauma and surgery \\ - Lung surface damage: \\ Trauma-for example, rib fractures \\ Iatrogenic-for example, attempted \\ central line insertion \\ Rupture of lung cysts \\ - Alveolar air leak: \\ Barotrauma \\ Blast injury \\ - Via diaphragmatic foramina from \\ peritoneal and retroperitoneal structures \\ - Via the head and neck
}

will then recoil away from the chest wall and a pneumothorax will be produced. ${ }^{1}$

Air can enter the pleural space in a variety of different ways that are summarised in box 1 . In most situations the passage of air will be limited either by the closure of the causative defect or by the equilibration of pleural and atmospheric pressures. Unfortunately the pressure within the pneumothorax may increase above atmospheric pressure. This occurs if the opening to the pleura acts like a valve allowing air to enter, but not to leave. It also occurs when the patient is subjected to positive pressure ventilation. The high pressure within the chest may then produce severe haemodynamic effects and the pneumothorax will be described as a tension pneumothorax.

On the intensive care unit pneumothorax is commonly caused by barotrauma associated with the ventilation of patients with adult respiratory distress syndrome (ARDS). It is therefore important to describe the mechanisms of barotrauma associated with ARDS.

ARDS is an inflammatory disease of the lung caused primarily by an abnormal immune response, commonly as a result of major trauma or infection. ${ }^{2}$ It results in pulmonary shunt, poorly compliant lungs, and pulmonary infiltrates. ARDS tends to cause more atelectasis and loss of lung volume in the posterior, gravity dependent areas of the lung. ${ }^{3}$ Mechanical ventilation is often essential in ARDS to maintain adequate oxygenation and remove the considerable work of breathing from the patient. Unfortunately ventilation may cause further damage, known as barotrauma. The initial process in barotrauma is the production of perivascular interstitial emphysema. ${ }^{4}$ When the pressure gradient between the alveoli and the interstitium exceeds a critical level alveoli rupture and air enters the interstitium. The 
pressure at which this occurs is determined by the degree of lung damage. This damage may be produced by the underlying disease process, the inflammation associated with ARDS or the use of excessive tidal volumes during ventilation. ${ }^{5}$ Some clinicians believe that positive end expiratory pressure may have some protective effect in preventing this damage, ${ }^{6}$ while any effects of hyperoxia remain unknown. ${ }^{7}$

Air escaping from ruptured alveoli then tracks proximally along the vascular sheaths and interlobular septa centrally to the hilum, resulting in a pneumomediastinum. Multiple areas of rupture must occur to produce clinically significant emphysema, however, once the process has occurred air will continue to move proximally into the mediastinum as long as the driving pressure gradient remains high. As well as this proximal movement, extraalveolar air may also form subpleural air cysts. ${ }^{8}$ These are most common along the anterior, medial, and inferior surfaces of the lung. Although normally a few millimetres across, these cysts may reach several centimetres in diameter. Rupture of either pleural air cysts or the mediastinal pleura will then result in a pneumothorax.

The over-distension of the non-dependent areas of the lung, and the role of the mediastinal pleura explain why anterior medial and subpulmonary pneumothoraces are more common in ARDS. ${ }^{9}$ As well as causing a pneumothorax, air in the mediastinum may also extend along perivascular connective tissue into the neck, retroperitoneum, peritoneum, and subcutaneous tissues. ${ }^{10}$ Subcutaneous emphysema is not directly harmful, however its detection on clinical or radiological examination is important as it suggests the lung has been subject to significant barotrauma. Once a pneumothorax has occurred, the high pressures generated during mechanical ventilation may easily cause the pneumothorax to tension and produce haemodynamic effects. Even with these high pressures the stiff, non-compliant nature of the lung and the pleural inflammation associated with ARDS may stop the surrounding lung from collapsing. A tension pneumothorax may therefore exist without total lung collapse or mediastinal shift. ${ }^{11}$

The proximal movement of air from ruptured alveoli towards the hilum is also an important mechanism in barotrauma caused by other disease processes, for example blast injury, positive pressure ventilation, and rapid deceleration associated with trauma.

\section{Diagnosis of pneumothorax}

The diagnosis of pneumothorax in critical illness is made from the history and examination of the patient and confirmed, where possible, by radiological investigation.

HISTORY

The factors that are important in the history relate to the underlying disease process and any potential for iatrogenic pneumothorax (box 2).

Outside intensive care practice the majority of pneumothoraces are idiopathic or associated
Box 2: Disease processes and iatrogenic procedures associated with pneumothorax

Disease processes

- ARDS

- Pneumonia:

Pneumocystis

Tuberculosis

Bacterial pneumonia

- Trauma

- Chronic obstructive lung disease

Iatrogenic procedures

- Positive pressure ventilation

- Attempted central line insertion

- Surgical procedures in the thorax, head, or neck

- Abdominal procedures using bowel or peritoneal distension

with chronic obstructive lung disease. ${ }^{12}$ Idiopathic pneumothoraces are associated with small areas of emphysema and cystic change, normally found at the apex of the lung. ${ }^{13}$ Any pneumonic process may also produce pneumothorax. The original descriptions of pneumothorax were commonly associated with tuberculosis. ${ }^{14}$ Pneumocystis is also frequently associated with pneumothorax ${ }^{15}$ as are more common bacterial pneumoniae.

Pneumothorax is also common after trauma. In a retrospective review of blunt thoracic trauma, pneumothorax was present in almost $20 \%$ of patients. ${ }^{16}$ The majority of patients with thoracic trauma are multiply injured, hence examination of these patients is difficult and cases of pneumothorax or haemothorax may not be diagnosed on initial assessment. ${ }^{17}$ Although pneumothorax may be caused by fractured ribs, it may also be present with an intact rib cage. ${ }^{16}$ In this situation pneumothorax is most commonly caused by alveolar air leak occurring during deceleration at the time of injury. Pneumothorax may also rarely be caused by tracheobronchial ${ }^{18}$ or oesophageal injury. ${ }^{19}$

\section{Iatrogenic factors}

The importance of barotrauma in relation to mechanical ventilation has already been described. Although any ventilated patient will be at risk, some factors in the history may point to an increased risk. These include the presence of ARDS, ${ }^{20}$ high peak airway pressures,${ }^{21}$ and previous pneumothorax. ${ }^{22}$ Unfortunately, even when lung protection strategies are used to reduce airway pressures there is still a significant incidence of pneumothorax, ${ }^{23}$ this has lead some authors to question the importance of high pressures or lung volumes in the development of barotrauma. ${ }^{20}$

As previously stated a pneumothorax is often present in patients with ARDS without the lung completely collapsing. If a chest drain is inserted into such a pneumothorax the drain 

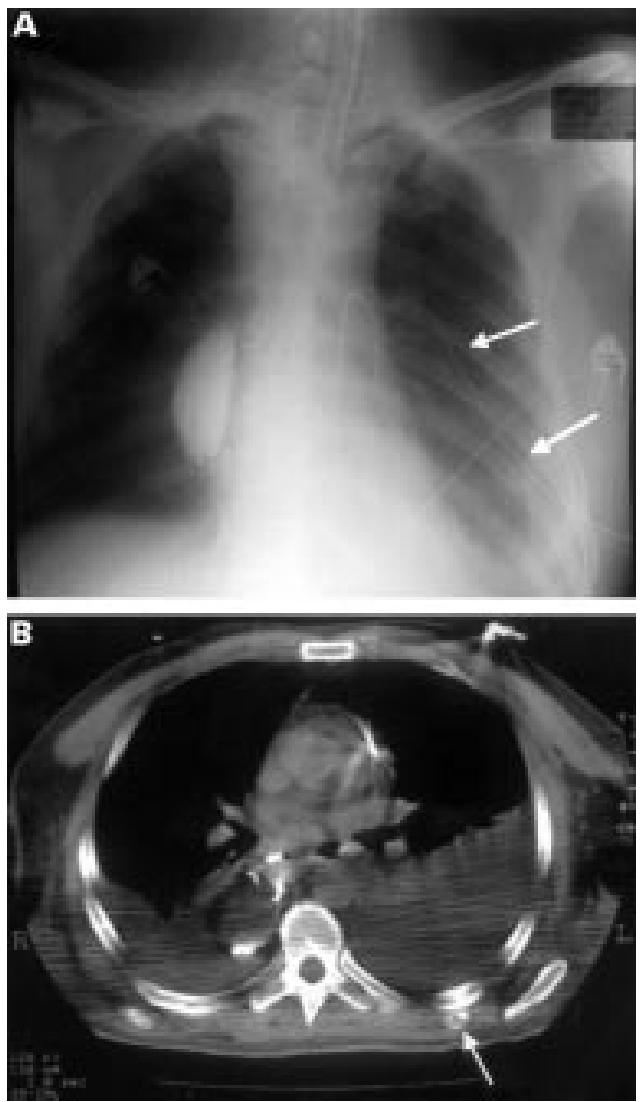

Figure 1 (A) The chest drain appears to be placed well into the chest on the frontal radiograph (arrows). A computed tomogram (B) shows that the drain is lying posterior to the chest wall having been tunnelled in the subcutaneous tissues.

may lie posteriorly behind the lung or within a lung fissure and can then be occluded by the heavy consolidated areas in the posterior portions of the lung. The presence of a chest drain does not therefore preclude the possibility of a recurrent pneumothorax on that side. In a description of this problem chest tubes misplaced in this way were found to be horizontal on frontal radiographs while correctly placed tubes ran laterally up the side of the chest wall. ${ }^{22}$ In patients with horizontal misplacement nine out of 20 pneumothoraces recurred, four under tension.

As well as malposition of a chest drain within the thoracic cavity it is also possible that drains, particularly those placed in suboptimal conditions, may not even enter the thoracic cavity. ${ }^{24}$ Figure 1 shows an example of this, the drain appears well placed on a frontal radiograph, but computed tomography shows it to have been placed in the subcutaneous tissues. As well as being malpositioned chest drains may also damage the lung parenchyma and hence be a cause of pneumothorax, even in the contralateral lung. ${ }^{25}$ The increased use of blunt dissection and the abandonment of the use of trochars for drain insertion should reduce this complication, ${ }^{26}$ however, a drain may still damage the underlying lung particularly if the lung is abnormal ${ }^{27}$ and may even enter the substance of the lung. ${ }^{25}$

Pneumothorax is a well recognised complication of other practical procedures. In a study
Box 3: Examination findings associated with pneumothorax and tension pneumothorax

Respiratory system

- Ventilator observations: reduction in tidal volume during pressure controlled ventilation. Increase in airway pressure with volume controlled ventilation.

- Chest signs: increased percussion note. Decreased breath sounds. Tracheal deviation away from the side of the pneumothorax.

- General examination: hypoxia, surgical emphysema.

- Examination of drainage systems: reduction or cessation of air leak through the system, blockage of a drain.

Cardiovascular system

- Tachycardia and hypotension.

- Increased pulmonary diastolic pressure and central venous pressure.

- Pulsus paradox.

- Drop in cardiac output.

of 1303 central line placements, pneumothorax occurred on five occasions. ${ }^{28}$ The incidence is sufficiently small after guidewire exchange of central lines for the routine ordering of chest radiography to have been questioned. ${ }^{29}$ Similarly, although pneumothorax is a recognised complication of percutaneous tracheostomy, ${ }^{30}$ its incidence is felt to be sufficiently small to question the value of a routine postprocedure chest radiography. ${ }^{31}$

The passage of air from the mediastinum into the neck, retroperitonium, and peritonium can be reversed if appropriate pressure gradients exist. For this reason laparoscopic procedures, particularly involving the oesophagus, may be a cause of pneumothorax. ${ }^{32}$ Pneumothorax may also be caused by dental procedures $^{33}$ and even colonoscopy. ${ }^{34}$ The pressure of gas used to distend the colon may cause gas to leak into the retroperitoneal space and hence into the mediastinum and pleural cavity. Similarly the use of compressed air in dental procedures may also result in pneumothorax. A recent history of any of these procedures as well as more obvious procedures in the thorax may therefore point to a diagnosis of pneumothorax.

\section{Examination}

Several findings in the examination of the respiratory and cardiovascular systems may help establish the diagnosis of pneumothorax and tension pneumothorax (box 3). It is important to note that these signs are all non-specific. The changes in ventilator observations, for example, could also be found with an obstruction to the endotracheal tube. The chest signs associated with pneumothorax are particularly difficult to interpret, for example collapse and consolidation on one side of the chest will cause increased percussion note on the other side of 


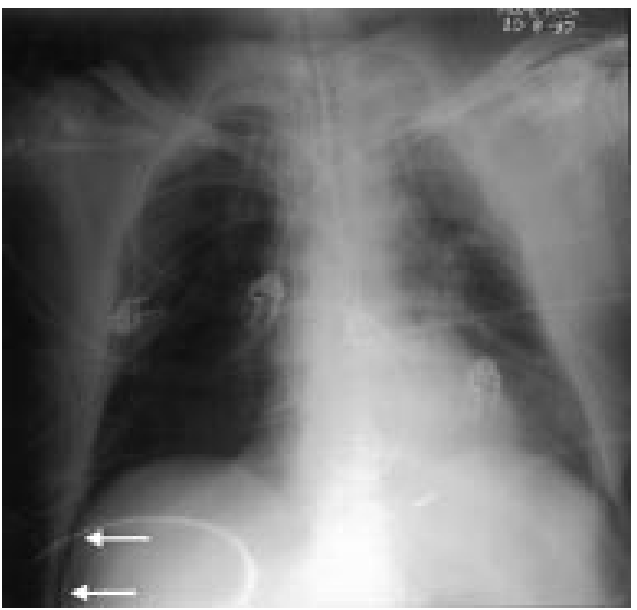

Figure 2 A pneumothorax in a supine patient. The deep sulcus sign (arrows). Compare with the costophrenic angle on the other side.

the chest and this may then be misinterpreted as a pneumothorax. As previously stated even a tension pneumothorax may exist in ARDS without complete collapse of the ipisilateral lung and even without ARDS a haemothorax or pneumothorax may easily be missed. Careful inspection and repeated auscultation of the chest, particularly in both mid-axillary lines, is therefore important. In addition to examining the patient and ventilator any chest drains should also be examined together with their drainage systems. The use of drainage systems is outside the scope of this article but has been well reviewed elsewhere. ${ }^{36}$

The haemodynamic changes associated with a tension pneumothorax are also quite nonspecific. The principle change in the pulmonary artery waveform is an elevation in the pulmonary artery diastolic pressure. ${ }^{37}$ The increase in thoracic pressure associated with the tension causes the alveolar pressure to rise above the pulmonary venous pressure. The pulmonary artery diastolic pressure is then determined by alveolar pressure, a situation similar to that described for West's zone 2 of the lung. ${ }^{38}$ Pressure within the chest will

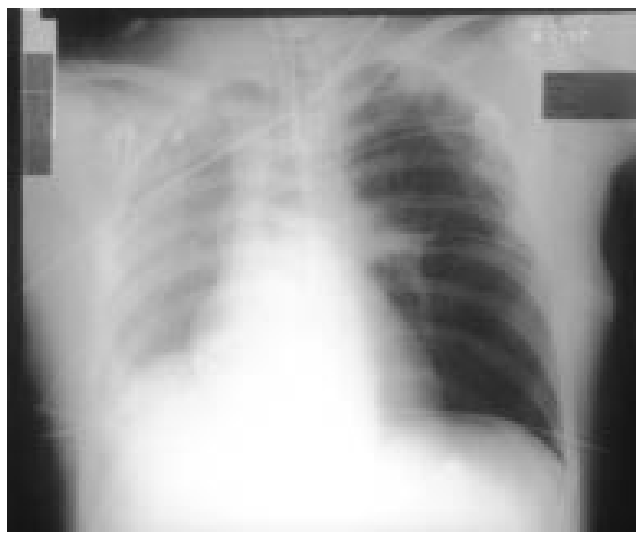

Figure 3 A right sided pneumothorax with underlying lung consolidation and pleural fluid. There are absent lung markings as a result of consolidation, and pleural fluid lying posteriorly contributes to the increased opacification of the hemithorax. A pneumothorax lying anteriorly in the chest causes a sharp outline to the mediastinum and right heart border. continue to fluctuate with the respiratory cycle. Hence a pulsus paradoxus may also be observed on the arterial trace.

Although non-specific, the association of respiratory and haemodynamic signs found with a tension pneumothorax are a medical emergency. Severe haemodynamic compromise will require urgent needle decompression of the pneumothorax before its diagnosis being confirmed radiologically. Fortunately this situation is uncommon and there is frequently time for radiological investigations to help establish the diagnosis of a simple pneumothorax.

\section{Radiological investigations}

The erect posteroanterior expiratory chest radiograph normally recommended for the investigation of pneumothorax is not practical in critical illness. The supine anteroposterior and lateral chest radiographs are frequently all that is available on the intensive care unit. Much more accurate information may also be obtained from thoracic computed tomograms in those patients well enough to be transported. ${ }^{39}$

With a patient in the supine position, large amounts of free air can collect in the anterior

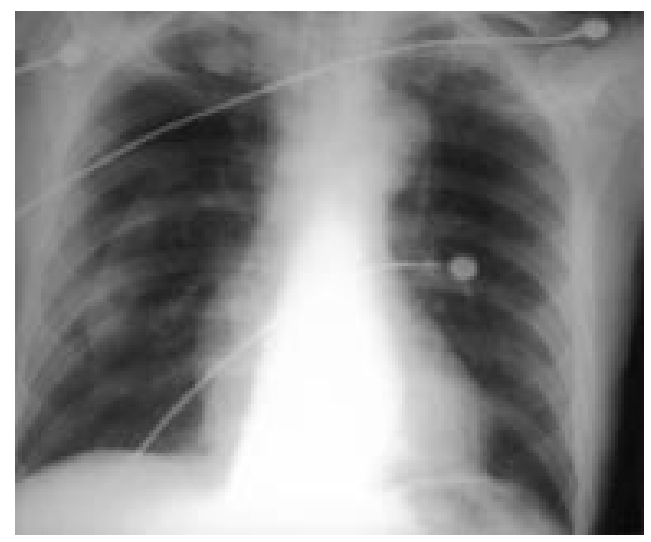

Figure 4 A skin fold over the right side of the chest. Note the broad nature of the opacification. Lung markings are visible beyond the edge of the opacification. This was mistaken for a pneumothorax and attempted aspiration caused a pneumothorax and surgical emphysema.

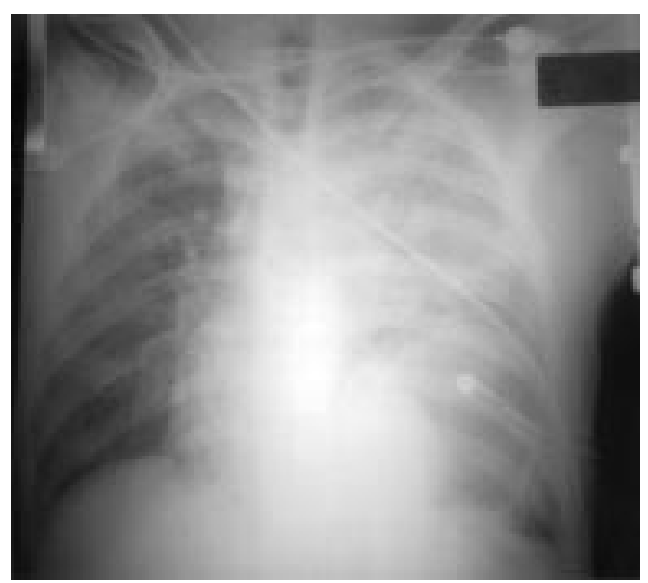

Figure 5 Stomach lying within the left side of the chest. A stab wound to the lower left chest has ruptured the diaphragm allowing the stomach to rise into the chest. Note the absence of a normal left hemidiaphragm. At surgery it was found that the chest drain had fortunately displaced rather than perforated the stomach. 

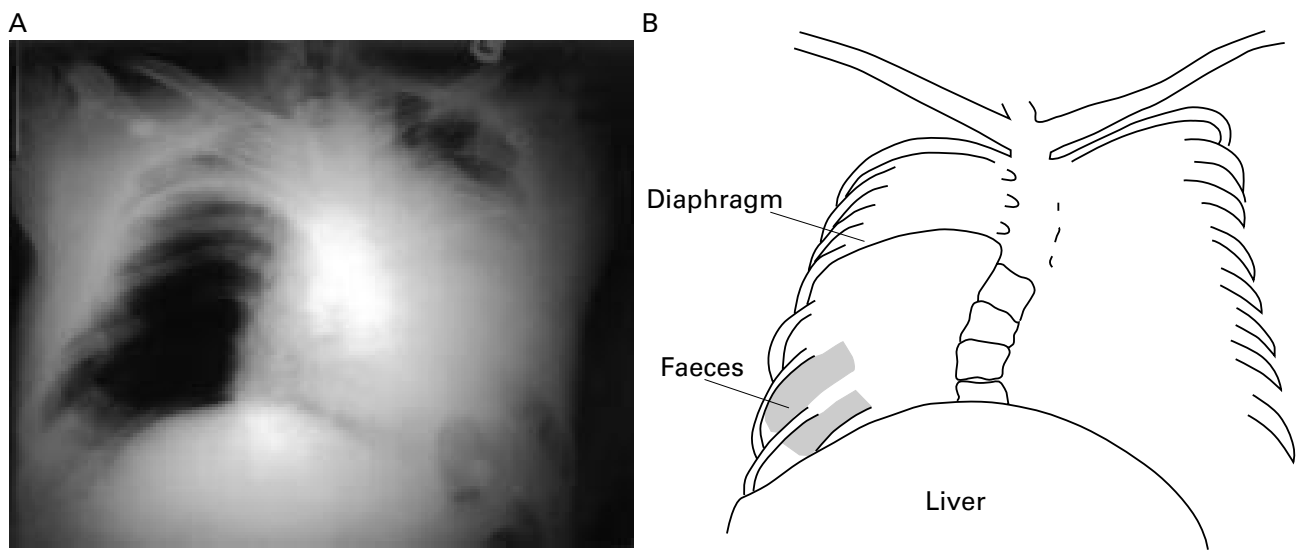

Figure 6 (A) Large bowel obstruction has caused a dilated loop of bowel to become interposed between the right hemidiaphragm and the liver. The position of the diaphragm (arrows) was not appreciated and a chest drain was inserted directly into large bowel; (B) line drawing of $(A)$.

part of the chest without the characteristic lung edge being visible (fig 2). The deep sulcus sign describes a costophrenic angle that extends more inferiorly than usual as a result of air lying in the costophrenic angle. ${ }^{40}$ If the patient does not have a bilateral pneumothorax it can be helpful to compare this with the normal side. On a normal chest radiograph the area of the liver is relatively opaque as the exposure is set to maximally visualise the low density lungs. When air collects in the costophrenic angle anteriorly over the liver, the liver will appear more radiolucent than usual. On the left, air will outline the medial aspect of the hemidiaphragm under the heart.

A pneumothorax commonly causes a radiolucent hemithorax with absent lung markings, however a pneumothorax can be present in a hemithorax that appears more radiopaque when there is underlying lung consolidation and pleural fluid (fig 3).

If there is doubt about the presence of a pneumothorax on a frontal supine radiograph, a film with the patient in a lateral decubitus position, with the affected side uppermost, can be helpful in demonstrating a lung edge. ${ }^{41}$

\section{Radiographs which may be confused with pneumothorax}

There are a number of situations where abnormal lucency or an apparent lung edge may be caused by abnormalities other than a pneumothorax. Abnormalities outside the thoracic cavity, abdominal contents within the chest, or abnormalities of the lung may cause this itself.

\section{SKIN FOLDS}

When a portable chest radiograph is performed, the $x$ ray cassette is positioned behind the patient and a fold of skin between the chest wall and the cassette can lead to a density on the radiograph, which can be mistaken for a pneumothorax. ${ }^{42} \mathrm{~A}$ pneumothorax gives rise to a thin pleural edge whereas a skin fold causes a broad opaque band, with lung markings still visible beyond the edge (fig 4). The fold may extend beyond the confines of the chest wall, which is conclusive proof of its nature. Tubes lying outside the patient can cause a line

\section{Learning points}

- Don't wait for a radiograph if there are clinical signs of a tension pneumothorax.

- A chest drain apparently well positioned on the radiograph may be lying in the soft tissues.

- The appearances of a pneumothorax on a supine radiograph are different from the classic appearances on an erect radiograph.

- Treat the patient not the radiograph. Don't act on a radiographic appearance if it does not fit the clinical picture. Get an expert opinion on the radiograph first.

projected over the periphery of the lung that must not be mistaken for a lung edge.

VISCERAL GAS WITHIN THE CHEST

Diaphragmatic hernia and diaphragmatic ruptures allow abdominal visceral contents into the chest. Air filled stomach and bowel entering the chest through these openings must therefore be distinguished from a pneumothorax. Normal bowel mucosal folds and an inability to define a normal contour to the diaphragm are clues (fig 5).

Dilated viscera and pneumoperitoneum may also displace an intact diaphragm high into the chest, and it is important to define the position of the diaphragm (fig 6).

\section{EMPHYSEMATOUS BULLAE}

The bullae of emphysema can be very large and when situated in the periphery of the lung can mimic a loculated pneumothorax. A chest drain inserted into a bullous in the mistaken belief that it is a pneumothorax is not uncommon. This is not surprising as emphysema is a known predisposing factor for a pneumothorax and patients with an exacerbation of their emphysema can present with a fairly sudden worsening of their breathlessness. The lack of a lung edge, the round nature of the bullous, and the presence of multiple bullae elsewhere in the lung are all clues to the diagnosis. In difficult 
cases computed tomography can be helpful in distinguishing between the two.

If in doubt about the diagnosis of a pneumothorax treat the patient and not the radiograph, and do not act on the appearances of a radiograph if it does not fit the clinical picture.

1 West JB. Mechanics of breathing. In: West JB. Respiratory physiology - the essentials. Baltimore: Williams and Williams, 1995: 89-116.

2 Luce M. Acute lung injury and the acute respiratory distress syndrome. Crit Care Med 1998;26:369-76.

3 Gattinoni L, Pelosi P, Pesenti A, et al. CT scan in ARDS clinical and physiopathological insights. Acta Anaesthesio Scand 1991;35: S95 87-96.

4 Macklin CC. Transport of air along sheaths of pulmonic blood vessels from alveoli to mediastinum. Arch Intern Med 1939;64:913-26.

5 Manning HL. Peak airway pressure: why the fuss. Chest 1994;105:242-7.

6 Fernandez-Mondejar E, Vazquez-Mata G. PEEP: more than just support? Intensive Care Medicine 1998;24:1-2.

7 Capellier G, Beuret P, Clement L, et al. Oxygen tolerance in patients with acute respiratory failure. Intensive Care Medicine 1998;24:422-8.

8 Albelda SM, Gefter WB, Kelley MA, et al. Ventilatorinduced subpleural air cysts: clinical, radiographic and

pathologic significance. Am Rev Respir Dis 1983;127:360-5. Tocino IM, Miller MH, Fairfax WR. Distribution of
pneumothorax in the supine and semirecumbent critically ill pneumothorax in the supine and sem.

10 Maunder RJ, Pierson DJ, Hudson LD. Subcutaneous and mediastinal emphysema pathophysiology, diagnosis, and management. Arch Intern Med 1984;144:1447-53.

11 Ross IB, Fleiszer DM, Brown RA. Localized tension pneumothorax in patients with adult respiratory distress syndrome. Can f Surg 1994;37:415-9.

12 George RB, Herbert SJ, Shames JM, et al. Pneumothorax complicating pulmonary emphysema. $\mathcal{F} A M A \quad 1975 ; 234$ 389-93

13 Lichter I, Gwynne JF. Spontaneous pneumothorax in young subjects. A clinical and pathological study. Thorax 1971;26: 409-17.

14 Palmer JP, Taft RB. Spontaneous pneumothorax. $\mathcal{F} A M A$ 1931;96:653-7.

15 Pastores SM, Garay SM, Naidich DP, et al. Review: pneumothorax in patients with AIDS-related Pneumocystis pneumothorax in patients with AIDS-related Pneum

16 Shorr RM, Crittenden M, Indeck M, et al. Blunt thoracic Shorr RM, Crittenden M, Indeck M, et al. Blunt thoracic
trauma. Analysis of 515 patients. Ann Surg 1987;206:200-5.

7 Chen SC, Markmann JF, Kauder DR, et al. Hemopneumothorax missed by auscultation in penetrating chest injury. $\mathcal{F}$ Trauma 1997;42:86-9.

18 Flynn AE, Thomas AN, Schecter WP. Acute tracheobronchial injury. F Trauma 1989;29:1326-30.

19 Layton TR, DiMarco RF, Pellegrini RV. Tracheoesophageal fistula from nonpentitrating trauma. F Trauma 1980;20: $802-5$.

20 Gammon RB, Shin MS, Groves RH Jr, et al. Clinical risk actors for pulmonary barotrauma: a multivariate analysis. Am $\mathcal{F}$ Resp Crit Care Med. 1995;152 (4 Pt 1):1235-40.

21 Petersen GW, Baier H. Incidence of pulmonary barotrauma in a medical ICU. Crit Care Med. 1983;11:67-9.

22 Heffner JE, McDonald J, Barbieri C. Recurrent pneumothoraces in ventilated patients despite ipsilateral chest tubes. Chest 1995;108:1053-8.

23 Stewart TE, Meade MO, Cook DJ, et al. Evaluation of a ventilation strategy to prevent barotrauma in patients at high
risk for acute respiratory distress syndrome. $N$ Engl $\mathcal{F}$ Med risk for acute respira

24 Baldt MM, Bankier AA, Germann PS, et al. Complications after emergency tube thoracostomy: assessment with CT Radiology 1995;195:539-43.

25 Gerard PS, Kaldawi E, Litani V, et al. Right-sided pneumothorax as a result of a left-sided chest tube. Chest 1993;103 $1602-3$.

26 Tomlinson MA, Treasure T. Insertion of a chest drain: how to do it. Br 7 Hosp Med 1997;58:248-52.

27 Wilson AJ, Krous HF. Lung perforation during chest tube placement in the stiff lung syndrome. F Pediatr Surg 1974;9: 213-16.

28 Yilmazlar A, Bilgin H, Korfali G, et al. Complications of 1303 central venous cannulations. $\mathcal{f} R$ Soc Med 1997;90 319-21.

29 Frassinelli P, Pasquale MD, Cipolle MD, et al. Utility of chest radiographs after guidewire exchanges of central chest radiographs after guidewire exchanges

30 Berrouschot J, Oeken J, Steiniger L, et al. Perioperative complications of percutaneous dilational tracheostomy. complications of percutaneous dilation

31 Tarnoff $M$, Moncure M, Jones F, et al. The value of routine posttracheostomy chest radiography. Chest 1998;113:1647-

32 Perez JE, Alberts WM, Mamel JJ. Delayed tension pneumothorax after laparoscopy. Surg Laparosc Endosc 1997;7:70-2.
33 Shackleford D, Casani J. Diffuse subcutaneous emphysema, pneumomediastinum, and pneumothorax afer dental extraction. Ann Emerg Med 1993;22:248-50.

34 Ho HC, Burchell S, Morris P, et al. Colon perforation, bilateral pneumothoraces, pneumopericardium, pneumomediastinum, and subcutaneous emphysema complicating endoscopic polypectomy: anatomic and management considerations. Am Surg 1996;62:770-4.

35 Webb T. Pneumothorax and pneumomediastinum during colonoscopy. Anaesth Intensive Care 1998;26:302-4.

36 Kam AC, O'Brien M, Kam PCA. Pleural drainage systems. Anaesthesia 1993;48:154-61.

37 Yu PY, Lee LW. Pulmonary artery pressures with tension pneumothorax. Can f Anaesth 1990;37:584-6.

38 West JB. Blood flow. In: West JB, ed. Respiratory physiologythe essentials. Baltimore: Williams and Williams, 1995: $89-116$.

39 Tagliabue M, Casella TC, Zincone GE, et al. CT and chest radiography in the evaluation of adult respiratory distress syndrome. Acta Radiol 1994;35:230-4.

40 Gordon R. The deep sulcus sign. Radiology 1980;136:257.3

41 Carr JJ, Reed JC, Choplin RH, et al. Plain and computed radiography for detecting experimentally induced pneumothorax in cadavers: implications for detection in patients. Radiology 1992;183:193-9.

42 Fisher JK. Skin fold versus pneumothorax. AfR Am f Roentenol 1978;130:791-2.

\section{Questions}

(1) Should a clinical diagnosis of pneumothorax always be confirmed radiologically?

No. Tension pneumothorax is a medical emergency and may require immediate needle decompression before radiological investigation.

(2) In what situations may a chest drain appear to be within the thorax on frontal radiograph when it is in fact malpositioned?

Such a drain may be in subcutaneous tissues, behind consolidated lung, in a lung fissure, or within the substance of the lung.

(3) What signs should be looked for on a frontal radiograph in a supine patient who is suspected of having a pneumothorax? What other radiological investigations may be used to confirm the diagnosis?

The “deep sulcus sign" describes a costophrenic angle that extends more inferiorly than usual as a result of air lying in the costophrenic angle. The liver appears more radiolucent than usual due to air lying anteriorly in the costophrenic angle, and on the left side, air will outline the medial aspect of the hemidiaphragm under the heart. A radiograph with the patient in a lateral decubitus position, with the affected side uppermost, can be helpful in demonstrating a lung edge. In patients well enough to be transported, thoracic computed tomography can be helpful in locating the position of a pneumothorax and accurately siting a chest drain.

(4) How may abnormalities that mimic a pneumothorax be classified?

They may be outside the chest, for example a skin fold, they may be due to abdominal viscera that are with in the chest, or they may be abnormalities arising from the lung itself.

(5) Has the use of lung protective strategies in ventilation been convincingly shown to reduce the incidence of barotrauma in ARDS?

No. In a randomised controlled study of 120 patients ${ }^{23}$ no benefit was demonstrated in such a strategy. 